\title{
Exsolving two-phase flow in oil wells
}

\author{
Victoria E. Pereira* ${ }^{\dagger}$, Andrew C. Fowler ${ }^{\dagger \dagger}$ \\ †OCIAM, Mathematical Institute, University of Oxford, Oxford, UK \\ ${ }^{\ddagger}$ MACSI, Department of Mathematics and Statistics, University of Limerick, Limerick, Ireland \\ (Received 00 Month 20xx; final version received 00 Month 20xx)
}

\begin{abstract}
Oil wells contain two-phase liquid and gas mixtures driven upwards due to a pressure gradient. In this paper, we study a two-fluid model for vertical upwelling flow, and explicitly account for the exsolution of the dissolved gas as the pressure decreases along the well. We find that the application of Henry's law for the dissolved gas concentration predicts a rapid transition to a foam, which runs counter to intuition. In order to study ways in which this rapid transition could be avoided, we examine rate limiting non-equilibrium dynamics by incorporating nucleation and bubble growth dynamics in the two-phase model.
\end{abstract}

Keywords: two-phase flow, exsolution, oil wells, nucleation.

\section{Introduction}

Two-phase flow is the simultaneous flow of two fluids in different physical states. These systems are ubiquitous in nature. Examples include geological systems (e.g. volcanoes) and biological systems (e.g. blood flow). Moreover, there are wide ranging industrial applications, examples of which range from food manufacturing to oil and gas production. This paper is concerned with exsolving two-phase flow through oil wells.

Hydrocarbons are organic compounds created deep underground. In liquid or gaseous form hydrocarbons travel upward through rock pores, driven by high pressures from below, until they reach the surface, or until they are trapped by impermeable rock above. The subsurface hydrocarbon stores are called reservoirs, and oil wells are pipes connecting reservoirs to the surface.

Reservoir fluid is made up of mixtures of liquid and gaseous hydrocarbons, and water. The flow through the production system is therefore inherently multiphase. A further complication arises from the many different hydrocarbon components. Lighter hydrocarbons, for example methane, account for the numerous components of the gaseous phase. Heavier hydrocarbons, such as pentane, make up the liquid oil phase. In this paper, we consider a two-phase flow consisting of liquid and gas phases; the liquid phase generally comprises both water and the mixture of hydrocarbons, and because the physical properties (including viscosity and density) of oil and water are much closer to each other than they are to those of the gas phase, we treat the liquid phase as a homogeneous medium.

Hydrocarbon extraction requires a complex production system, both below and above ground. Multiple wells will be drilled down to the reservoir level. High pressures at the base and low pressures at the surface result in the reservoir fluid travelling up through the wells. This primary recovery driven by the initial reservoir pressure is known as natural lift. Over time, due to production, the reservoir pressure will decrease and the pressure gradient will tend towards hydrostatic equilibrium which results in reduced flow into the well. The flow can be encouraged by means of artificial lift systems which aim to increase the local energy

*Corresponding author. Email: pereira@maths.ox.ac.uk 
in the well (Golan and Whitson 1991), and secondary recovery methods aimed at increasing the reservoir pressure (Muggeridge et al. 2014).

There is clearly a two way coupling between the flow in the reservoir and the flow in the wells, and for a single reservoir there will be several injection and production wells. The production system therefore quickly becomes very complicated to analyse. Consequently, industrial models are based on nodal analysis, which break down the large system into components connected at nodes (Dale 1991).

Models of the reservoir predict the flow through a large porous domain, often kilometres in diameter. These supply boundary conditions to the near well bore domain, which is the linking component between the reservoir and the wells. Near well bore models analyse the porous flow in a radial domain localised around the well. The near well bore model feeds into the well model, which can be considered as a multiphase pipe flow. The top of the well links to the choke which is a restricted flow system that regulates the pressure and connects the subsurface to the surface facilities.

Well models are used to understand the multiphase flow through the well with the aim of optimising the well deliverability; to maximise flow rate whilst minimising the pressure under given reservoir and surface constraints. These models differ in how closely the derivation relies on fundamental laws of physics, and also whether they are steady in time or transient. Models are typically categorised as 'empirical' where constructed from data, and 'physics-based' where derived from laws of physics. In practice, it is common for a combination of empirical and physics-based methods to be used.

In the past, the common practice in the industry was to use empirical models known as correlations like those by Poettman and Carpenter (1952), Hagedorn and Brown (1965), Duns Jr and Ros (1963) and Beggs and Brill (1973). These steady-state models are constructed from data collected from a particular well or artificial set-up. They are commonly used to determine steady-state flow and advise how to optimise the steady production. However, applicability of these empirical models is greatly limited as the results are reliable only for the specific system from which the data was collected. Accuracy can also be a problem, as these models are not governed by the laws of physics but are fitted to experimental data, the validity of which may be questionable.

In more recent years, there has been a move towards 'physics-based' modelling of oil wells (Shippen and Bailey 2012). Transient models, such as those by Livescu et al. (2010) and Bendiksen et al. (1991), are mainly used in the initial start-up of the well and when changes are being made to the system, for example the introduction of an artificial lift system. For the majority of the lifetime of the well, the dynamics are assumed to be at steady state, and thus steady-state models like those derived by Taitel and Dukler (1976), Ansari et al. (1990), and Cazarez-Candia and Vásquez-Cruz (2005) are utilised.

\subsection{Problem statement}

At the high pressures in the reservoir, different hydrocarbon components may be dissolved in the liquid phase. As the fluid mixture travels up the well the pressure decreases, allowing for the exsolution of the dissolved components. There is a pressure $p_{b}$, known as the bubblepoint pressure, at which gas is first released from the liquid (Economides 2013, Guo 2011). For pressures above the bubble-point, only liquid is present, and there is no free gas in the reservoir. At pressures below the bubble-point, gas and liquid phases co-exist.

We note the analogy of two-phase flow in an oil well with that in boilers and volcanoes. In boiling systems, the flow is heated to boiling point by an external wall heating. This causes nucleation of gas bubbles as steam is produced. A gas phase is also released in a volcano due to exsolution from the liquid magma.

In this paper, we propose a mathematical model for the exsolving two-phase flow in an oil 
well producing under natural lift. We consider a system with a liquid inflow at the bottom of the well where $p>p_{b}$. Exsolution is assumed to first occur at a point along the well where $p=p_{b}$. We consider parameter ranges for the oil viscosity and density appropriate for a light oil, such as that produced in the Ekofisk oil field in the North Sea (Van Den Bark and Thomas 1981).

The gaseous phase is clearly compressible, however, for the purposes of this paper we will neglect the compressibility of the gas. The oil in the liquid phase is also only slightly compressible; we therefore make the simplifying assumption that the density and viscosity of the oil, and consequently the liquid phase, remains constant throughout the domain. Whilst one might suppose that the highly compressible nature of the gaseous phase is important in this system, it can be shown that the inclusion of compressibility for the gas phase does not significantly affect the results, see Pereira (2019). For these reasons, our presentation assumes incompressibility of both phases.

The model is motivated by recent work by Fowler and Robinson (2018) for the exsolving flow in volcanic conduits. We will follow the derivation for the oil-well application. As discussed above, there is plentiful research concerning the modelling of multiphase flow in oil wells. To our knowledge, previous models of oil wells account for exsolution through mass transfer between the phases, but do not account for the conservation of the dissolved gas phase. We use a two-fluid modelling approach similar to that of Drew and Passman (2006).

\section{Two-fluid model with exsolution dynamics}

In this section we introduce a two-fluid model for the isothermal exsolving upwelling flow in an oil well of length $L$ and diameter $d$. The assumption of constant temperature is a reasonable simplification, as the temperature change along the well is relatively small ((Ghorbani et al. 2018) give an estimate for the temperature change of $60^{\circ} \mathrm{K}$ ). Inclusion of temperature would simply introduce a mild height dependence in the gas equation of state. For an oil well we assume a one-dimensional domain as the production tubing is long and thin: the lengths are of the order of kilometres, and the diameter is typically $10 \mathrm{~cm}$. We describe the one-dimensional flow by the spatial coordinate along the pipe axis $z: z=0$ is the bottom of the pipe, and $z=L$ the top.

The two-fluid model presented by Drew and Passman (2006) needs to be adapted to include the concentration of dissolved gas in the liquid. We will follow the derivation by Fowler and Robinson (2018).

Conservation of mass for the two phases is given by

$$
\begin{aligned}
\left(\alpha \rho_{g}\right)_{t}+\left(\alpha \rho_{g} v\right)_{z} & =\Gamma, \\
{\left[(1-\alpha) \rho_{l}\right]_{t}+\left[(1-\alpha) \rho_{l} u\right]_{z} } & =-\Gamma,
\end{aligned}
$$

where we have used subscripts $t$ and $z$ to denote partial derivatives with respect to time and the spatial coordinate $z ; \alpha$ is the gas volume fraction, $\rho_{g}$ is the gas density, $\rho_{l}$ is the liquid density, $v$ is the velocity of the gas phase, $u$ is the liquid velocity and $\Gamma$ is the exsolution rate. We assume the density of the liquid phase to be the average of the oil and water densities; $\rho_{l}=\frac{1}{2}\left(\rho_{w}+\rho_{o}\right)$, where $\rho_{w}$ and $\rho_{o}$ are the densities of water and oil respectively. Note that this assumes equal amounts of water and oil in the liquid phase. Conservation of mass for the solute component gives

$$
\left[(1-\alpha) \rho_{l} c+\alpha \rho_{g}\right]_{t}+\left[(1-\alpha) \rho_{l} c u+\alpha \rho_{g} v\right]_{z}=0,
$$

where $c$ is concentration of dissolved gas defined as a mass fraction. We can use (2.1a) to 
rewrite this as

$$
\left[(1-\alpha) \rho_{l} c\right]_{t}+\left[(1-\alpha) \rho_{l} c u\right]_{z}=-\Gamma .
$$

The momentum-conservation equations for the liquid and gas phases are

$$
\begin{aligned}
\left(\alpha \rho_{g} v\right)_{t}+\left(\alpha \rho_{g} v^{2}\right)_{z} & =-\left(\alpha p_{g}\right)_{z}-\alpha \rho_{g} g+M_{g}, \\
{\left[(1-\alpha) \rho_{l} u\right]_{t}+\left[(1-\alpha) \rho_{l} u^{2}\right]_{z} } & =-\left[(1-\alpha) p_{l}\right]_{z}-\tau_{l}^{w}-(1-\alpha) \rho_{l} g+M_{l},
\end{aligned}
$$

where $p_{g}, p_{l}$ are the gas and liquid pressures, $g$ is the acceleration due to gravity, $\tau_{l}^{w}$ is the resistance due to wall shear stress for the liquid phase, and $M_{g}, M_{l}$ are the rates of interfacial momentum transfer. Note that we have neglected the viscous stress tensors for both phases. Furthermore, we have assumed the wall stress of the gas to be negligible, as we expect the gas phase to initially appear as bubbles dispersed in the liquid phase. Note that this assumption will need to be altered if $\alpha$ becomes too large causing a bubbly-to-slug flow régime transition; the consequences of which will be considered later in section 5 . The liquid wall stress is given (assuming $u>0$ ) by

$$
\tau_{l}^{w}=\frac{4 f_{l} \rho_{l} u^{2}}{d},
$$

where $f_{l}$ is the friction factor, and $d$ is the tube diameter.

The interfacial momentum transfer terms account for the interfacial pressure and force densities (Ishii and Mishima 1984):

$$
\begin{array}{r}
M_{g}=p_{g i} \alpha_{z}-F_{D}-F_{v m}, \\
M_{l}=-p_{l i} \alpha_{z}+F_{D}+F_{v m},
\end{array}
$$

where $p_{l i}, p_{g i}$ are interfacial pressures, and $F_{D}$ and $F_{v m}$ denote the drag and virtual-mass forces. We have assumed negligible interfacial flux and interfacial stress motivated by an expected dispersed bubbly flow, at least initially. The drag and virtual mass force densities are

$$
\begin{aligned}
F_{D} & =\frac{3}{8} \frac{C_{D} \alpha \rho_{l}}{r_{b}}(v-u)|v-u|, \\
F_{v m} & =\frac{1}{2} \alpha_{g} \rho_{l}\left[\left(u_{t}+u u_{z}\right)-\left(v_{t}+v v_{z}\right)\right],
\end{aligned}
$$

where $C_{D}$ is the drag coefficient, $r_{b}$ is the typical bubble radius.

If we assume that the surface energy effects are small enough to take $p_{k i}=p_{k}$, where the subscript $k$ denotes the $k^{\text {th }}$ phase, in (2.6a)-(2.6b), and use the definition for the wall stress in (2.5), then (2.4a) and (2.4b) become

$$
\begin{aligned}
\left(\alpha \rho_{g} v\right)_{t}+\left(\alpha \rho_{g} v^{2}\right)_{z}=- & \alpha p_{g z}-g \alpha \rho_{g}-\frac{3}{8} \frac{C_{D} \alpha \rho_{l}}{r_{b}}|v-u|(v-u) \\
& -\frac{1}{2} \alpha_{g} \rho_{l}\left[\left(u_{t}+u u_{z}\right)-\left(v_{t}+v v_{z}\right)\right], \\
{\left[(1-\alpha) \rho_{l} u\right]_{t}+\left[(1-\alpha) \rho_{l} u^{2}\right]_{z}=- } & (1-\alpha) p_{l z}-\frac{4 f_{l} \rho_{l} u^{2}}{d}-g(1-\alpha) \rho_{l} \\
& +\frac{3}{8} \frac{C_{D} \alpha \rho_{l}}{r_{b}}|v-u|(v-u)+\frac{1}{2} \alpha_{g} \rho_{l}\left[\left(u_{t}+u u_{z}\right)-\left(v_{t}+v v_{z}\right)\right] .
\end{aligned}
$$

To close the system of model equations we require equations of state for the densities $\rho_{g}, \rho_{l}$. As discussed above, we will assume both phases to be incompressible, and consequently take $\rho_{g}$ and $\rho_{l}$ to be fixed constants. 
An additional closure relation comes from prescribing the pressure difference between the phases. We rely on previous work by Stuhmiller (1977) and Pauchon and Banerjee (1986) which examined the potential flow around a spherical bubble of constant radius to prescribe a pressure difference dependent on the relative velocity:

$$
p_{g}-p_{l}=-H \rho_{l}(v-u)^{2},
$$

where $H=\frac{1}{4}$ represents the effect of Reynolds stress and bubble induced distortion to the streamlines of the liquid phase (Lisseter and Fowler 1992).

It is well understood through Henry's law that the concentration of dissolved gas is dependent on the pressure in the system; $c=K p_{l}^{\nu}$, where $K$ is a function of temperature $T$ and $\nu$ is an empirically calculated constant. In an isothermal flow the value $K$ is an assumed constant. A commonly used correlation for oil developed by Vazquez and Beggs (1977) gives $\nu \approx 1$. The equilibrium between the dissolved gas concentration and the liquid pressure is therefore assumed to be:

$$
c=K p_{l} .
$$

Recall that the pressure at which the dissolved gas starts to come out of solution is known as the bubble-point pressure $p_{b}$. Thus at this pressure the concentration of dissolved gas is saturated and we have: $c_{0}=K p_{b}$, where $c_{0}$ is the initial concentration of dissolved gas. If we assume the values for $p_{b}$ and $c_{0}$ to be known, we can calculate the value of the parameter $K$.

Henry's law (2.10) states that the amount of dissolved gas descreases with the pressure in the well, thus as the pressure drops more gas comes out of solution. In reality, growth of bubbles occurs through diffusion of the dissolved gas phase to the bubble surface; this may be a rate limiting mechanism for gas exsolution, and we examine this further in section 4 .

The model equations $(2.1 \mathrm{a}),(2.1 \mathrm{~b}),(2.3),(2.8 \mathrm{a}),(2.8 \mathrm{~b}),(2.9),(2.10)$ are to be solved for the unknowns $\alpha, c, v, u, p_{g}, p_{l}, \Gamma$.

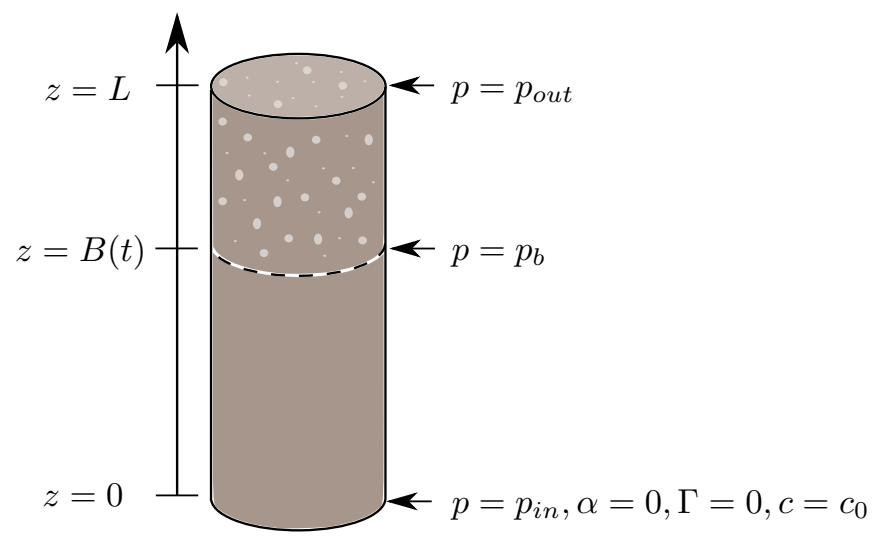

Figure 1.: Oil well domain with boundary conditions.

\subsection{Boundary conditions}

We consider a system with an inflow of liquid with an initial concentration $c_{0}$ of dissolved gas at the base $z=0$. The inlet pressure is denoted $p_{i n}$ and the inlet velocity is $U(t)$; thus the inlet boundary conditions are

$$
p_{l}=p_{\text {in }}, \quad \alpha=0, \quad c=c_{0}, \quad u=U(t) \quad \text { at } \quad z=0 .
$$

We prescribe the inflow velocity by consideration of the reservoir domain in which the producing well can effectively be considered as a point sink. Flow into the well is assumed to 
be as radial flow, we denote the radial coordinate $r$, through a porous medium driven by the pressure difference $\left(p_{\text {res }}-p_{\text {in }}\right)$ between the far-field reservoir pressure, $p_{\text {res }}$ and that at the bottom of the well. This causes an approximate pressure field

$$
p_{l} \sim p_{\text {res }}-\frac{d\left(p_{\text {res }}-p_{\text {in }}\right)}{2 r},
$$

(the quantity $d / 2$ is the pipe radius and also the effective radius of the spherical sink for the reservoir flow), and thus a volume flux $2 \pi k\left(p_{\text {res }}-p_{\text {in }}\right) d / \mu$, where $k$ is permeability and $\mu$ is liquid viscosity. Equating this to the volume flux in the well gives the prescription for the inlet velocity,

$$
U(t)=\frac{8 k\left(p_{\text {res }}-p_{\text {in }}\right)}{\mu d} .
$$

Gas starts to come out of solution when the pressure reaches the bubble-point pressure $p_{b}$, whose location is denoted $z=B(t)$. The domain is thus split into a single-phase liquid domain in $0 \leq z \leq B(t)$ and a two-phase domain in $B(t) \leq z \leq L$, see figure 1. On the bubble-point boundary, the boundary conditions are

$$
p_{l}=p_{b}, \quad \alpha=0, \quad c=c_{0}, \quad u=U(t) \text { at } z=B(t),
$$

the last being a consequence of the incompressibility of the liquid.

Finally, at the exit, we prescribe the pressure; this is known as the 'choke' pressure, and is used to control the flow in the well:

$$
p_{l}=p_{g}=p_{\text {out }} \text { at } z=L .
$$

\subsection{Dimensionless model}

To non-dimensionalise the model equations we introduce the following scalings:

$$
z \sim L, \quad t \sim \frac{L}{U_{0}}, \quad v, u, U \sim U_{0}=\frac{8 k p_{\text {res }}}{\mu d}, \quad c \sim c_{0}, \quad \Gamma \sim \frac{\rho_{l} U_{0}}{L}, \quad p_{l}, p_{g} \sim \rho_{l} g L,
$$

where $U_{0}, c_{0}$ are typical velocity and dissolved gas concentration scales, respectively, and we suppose

$$
p_{\text {res }}=\rho_{c} g L,
$$

where $\rho_{c}$ is the density of crustal rock. Using these in the model equations (2.1a), (2.1b), (2.3), (2.8a), (2.8b), (2.9) and (2.10) yields

$$
\begin{aligned}
\delta\left[\alpha_{t}+(\alpha v)_{z}\right] & =\Gamma, \\
-\alpha_{t}+((1-\alpha) u)_{z} & =-\Gamma, \\
{[(1-\alpha) c]_{t}+[(1-\alpha) u c]_{z} } & =-\frac{1}{c_{0}} \Gamma, \\
F^{2}\left\{\Gamma v+\delta \alpha\left(v_{t}+v v_{z}\right)\right\} & =-\alpha p_{g z}-\delta \alpha-\kappa_{D} \alpha|v-u|(v-u) \\
& +\kappa_{v m} f_{v m}, \\
F^{2}\left\{-\Gamma u+(1-\alpha)\left(u_{t}+u u_{z}\right)\right\} & =-(1-\alpha) p_{l z}-\kappa_{w} u^{2}-(1-\alpha) \\
p_{g}-p_{l} & =-H F^{2}(v-u)^{2}, \\
c & =\frac{p_{l}}{\Pi},
\end{aligned}
$$


where we have introduced the following dimensionless parameters,

$$
\begin{gathered}
F=\frac{U_{0}}{\sqrt{g L}}, \quad \delta=\frac{\rho_{g}}{\rho_{l}}, \quad \kappa_{D}=\frac{3 C_{D}}{8} \frac{U_{0}^{2}}{g r_{b}}, \quad \kappa_{v m}=\frac{1}{2} \frac{U_{0}^{2} \rho_{l}}{L}, \\
\kappa_{w}=\frac{4 f_{l} U_{0}^{2}}{g d}, \quad \Pi=\frac{c_{0}}{K \rho_{l} g L},
\end{gathered}
$$

and the dimensionless virtual-mass force:

$$
f_{v m}=\alpha_{g}\left[\left(u_{t}+u u_{z}\right)-\left(v_{t}+v v_{z}\right)\right] .
$$

Recall that from (2.10) we have $p_{b}=c_{0} / K$, so the parameter $\Pi$ is the dimensionless bubblepoint pressure. Note that for exsolution in the well we require that the bubble-point pressure is less than the reservoir pressure, i. e. $\Pi<p_{R}$ where $p_{R}=p_{\text {res }} / \rho_{l} g L$ is the dimensionless reservoir pressure.

\begin{tabular}{|c|l|l|}
\hline Parameter & Value & Source \\
\hline$C_{D}$ & $O(1)$ & Ishii and Mishima (1984) \\
$d$ & $0.1 \mathrm{~m}$ & Govier and Aziz (2008) \\
$f_{l}$ & 0.005 & Wallis (1969) \\
$k$ & $10^{-12}-10^{-15} \mathrm{~m}^{2}$ & Chen $(2007)$ \\
$L$ & $10^{3} \mathrm{~m}$ & Govier and Aziz $(2008)$ \\
$p_{\text {res }}$ & $28 \mathrm{MPa}$ & $\left(=\rho_{c} g L\right)$ \\
$U_{0}$ & $0.2 \mathrm{~m} \mathrm{~s}^{-1}$ & $\left(=8 k p_{\text {res }} / \mu d\right.$, using $\left.k=10^{-13} \mathrm{~m}^{2}\right)$ \\
$\mu$ & $10^{-4}-10^{-3} \mathrm{~Pa} \mathrm{~s}$ & Chen $(2007)$ \\
$\rho_{g}$ & $1.14 \mathrm{~kg} \mathrm{~m}^{-3}$ & Shoham $(2006)$ \\
$\rho_{w}$ & $10^{3} \mathrm{~kg} \mathrm{~m}^{-3}$ & Livescu et al. $(2010)$ \\
$\rho_{o}$ & $\leq 0.92 \times 10^{3} \mathrm{~kg} \mathrm{~m}^{-3}$ & Meyer and Attanasi $(2003)$ \\
$\rho_{l}$ & $0.96 \times 10^{3} \mathrm{~kg} \mathrm{~m}^{-3}$ & $\left(=\frac{1}{2}\left(\rho_{w}+\rho_{o}\right)\right)$ \\
$\rho_{c}$ & $2.8 \times 10^{3} \mathrm{~kg} \mathrm{~m}^{-3}$ & Christensen and Mooney (1995) \\
\hline
\end{tabular}

Table 1.: Parameters and typical values. The drag coefficient $C_{D}$ is a decreasing function of particle Reynolds number $R e_{P}=r_{b} U_{0} \rho_{l} / \mu$. In this paper, we assume the parameter values for a light oil with viscosity $\mu=10^{-3} \mathrm{~Pa} \mathrm{~s}$, and density $\rho_{l}=0.96 \times 10^{3} \mathrm{~kg} \mathrm{~m}^{-3}$.

The Péclet number is a measure of the size of thermal advection in comparison to heat transfer across the tube. The appropriate definition for a turbulent flow is

$$
\mathrm{Pe}_{T}=\frac{d}{f_{l} L} \approx 0.02,
$$

where we assume an eddy thermal diffusivity $\kappa_{T}=f_{l} U_{0} d$ and use the values in table 1 . This suggests that the oil temperature in the tube will simply follow the geothermal temperature, and as stated earlier, the variation is not large.

The boundary conditions (2.11), (2.14) and (2.15) become, respectively,

$$
\begin{array}{r}
p_{l}=p_{0}, \quad \alpha=0, \quad c=1, \quad u=U(t)=1-\delta_{c} p_{0} \quad \text { at } z=0, \\
p_{l}=\Pi, \quad \alpha=0, \quad c=1, \quad u=U(t) \text { at } z=b(t), \\
p_{l}=p_{g}=p_{1} \quad \text { at } z=1,
\end{array}
$$

where

$$
\delta_{c}=\frac{\rho_{l}}{\rho_{c}}, \quad p_{0}=\frac{p_{\text {in }}}{\rho_{l} g L}, \quad p_{1}=\frac{p_{\text {out }}}{\rho_{l} g L}, \quad b(t)=\frac{B(t)}{L} .
$$




\begin{tabular}{|c|c|}
\hline Parameter & Value \\
\hline$F$ & $2 \times 10^{-3}$ \\
$\delta_{c}$ & 0.34 \\
$\delta$ & $1.2 \times 10^{-3}$ \\
$\kappa_{D}$ & $\left(1.5 \times 10^{-3}\right) C_{D} /\left[r_{b}\right]$ \\
$\kappa_{v m}$ & $1.9 \times 10^{-2}$ \\
$\kappa_{w}$ & $0.8 \times 10^{-3}$ \\
$p_{R}$ & 2.97 \\
$\Pi$ & $<p_{R}$ \\
\hline
\end{tabular}

Table 2.: Dimensionless parameters and their approximate values using table $1 .\left[r_{b}\right]$ is the numerical value of $r_{b}$ in centimetres.

The model parameters are given in table 1 . The chosen values are used to compute the dimensionless parameters in table 2 . The velocity scale was determined from (2.13). Assuming $H=\frac{1}{4}$ in (2.18f) suggests we can take $p_{g} \approx p_{l}$, and denote the single pressure as $p$.

If we neglect terms of $O\left(F^{2}\right), O(\delta), O\left(\kappa_{w}\right)$ and $O\left(\kappa_{v m}\right)$ in $(2.18 \mathrm{~d})-(2.18 \mathrm{e})$, we get

$$
\begin{aligned}
\alpha p_{z} & =-\kappa_{D} \alpha|v-u|(v-u), \\
(1-\alpha) p_{z} & =-(1-\alpha)+\kappa_{D} \alpha|v-u|(v-u) .
\end{aligned}
$$

Adding the above two equations together shows that the pressure gradient is dependent on the gravitational deceleration:

$$
p_{z}=-(1-\alpha) \text {. }
$$

Using this in (2.24a) we find

$$
v-u=\sqrt{\frac{(1-\alpha)}{\kappa_{D}} .}
$$

If $\kappa_{D} \gg 1$, which is the case for sufficiently small bubbles, then $v \approx u$ and a homogeneous model is appropriate. We now proceed with this assumption.

\section{Homogeneous model}

The homogeneous model is that derived above, except that one writes the momentum equations in the form (2.25) and (2.26) and then replaces the second of these with the approximation $v=u$. Again we neglect terms of $O\left(F^{2}\right)$ and $O(\delta)$ in the momentum equation, and additionally the wall friction term $O\left(\kappa_{w}\right)$ and virtual mass forces $O\left(\kappa_{v m}\right)$. We then have

$$
\begin{aligned}
\delta\left[\alpha_{t}+(\alpha u)_{z}\right] & =\Gamma, \\
(1-\alpha)_{t}+[(1-\alpha) u]_{z} & =-\Gamma, \\
{[(1-\alpha) c]_{t}+[(1-\alpha) c u]_{z} } & =-\frac{\Gamma}{c_{0}}, \\
p_{z} & =-(1-\alpha), \\
c & =\frac{p}{\Pi} .
\end{aligned}
$$

These equations apply below the bubble-point height in the single-phase region providing we take $c=1$ if $p>\Pi$; the boundary conditions are those in $(2.22 \mathrm{a})-(2.22 \mathrm{c})$. We have retained 
the term in $\delta$ in (3.1a) as neglecting this is a singular approximation.

We note that there is a large range of possible parameter choices across the different kinds of oil and gases; on the scale of a single oil field there will be a range of possible parameters. Ghorbani et al. (2018) supplied data for 127 oil and gas production wells in the Reshadat oil field in Iran: oil viscosity $\mu_{o}=3.8-4.3 \times 10^{-3} \mathrm{~Pa} \mathrm{~s}$; oil density $\rho_{o}=0.9-0.94 \times 10^{3} \mathrm{~kg}$ $\mathrm{m}^{-3}$; reservoir pressure $p_{\text {res }}=17-26 \mathrm{MPa}$; bubble-pressure $p_{b}=14-18 \mathrm{MPa}$; well head pressure $p_{\text {out }}=2-6 \mathrm{MPa}$; and the gas-liquid ratio $G L R=7-137 \mathrm{~m}^{3} \mathrm{~m}^{-3}$ given at standard conditions, that is at atmospheric pressure $p_{a t m}=0.1 \mathrm{MPa}$ and $288 \mathrm{~K}$ temperature.

The GLR can be used to derive a mass fraction for the gas phase. Assuming $\rho_{g}=1 \mathrm{~kg} \mathrm{~m}^{-3}$ at standard conditions then, using the range $G L R=7-137$, the mass of the gas lies in the range $m_{g}=7-137 \mathrm{~kg}$. Similarly, using $\rho_{l}$ from table 1, we calculate the mass of the liquid phase to be $m_{l}=0.96 \times 10^{3} \mathrm{~kg} \mathrm{~m}^{-3}$. If we assume all the gas is initally dissolved in the liquid phase, we can derive a range for the initial dissolved gas concentration to be $c_{0} \approx 0.007-0.14$. We therefore study the system (3.1a)-(3.1e) in two limits: $\delta \ll 1, c_{0} \sim O(1)$ and $\delta \sim c_{0} \ll 1$.

\subsection{The case $\delta \ll 1, c_{0} \sim O(1)$}

In the single-phase region $0 \leq z \leq b(t), \alpha=0, c=1$ and $\Gamma=0$. (3.1b) then implies $u=U(t)$, as mentioned earlier. (3.1d) yields a linear pressure drop:

$$
p=p_{0}-z .
$$

Since $z=b(t)$ is defined by the bubble-point pressure $p=\Pi$, we have

$$
b(t)=p_{0}-\Pi .
$$

In the region $b(t) \leq z \leq 1$, we still have $\Gamma \approx 0$ from (3.1a). Using this together with (3.1e) in $(3.1 \mathrm{c})$ gives

$$
(1-\alpha)\left[p_{t}+u p_{z}\right]=0,
$$

which implies that above $z=b$, either $\alpha=1$ or the pressure stays constant. But from (3.1d) we have

$$
p_{z}=-(1-\alpha) \text {. }
$$

The two equations (3.4) and (3.5) demand that $\alpha=1$ and $p=\Pi$, which means that above $z=$ $b(t)$ the pressure stays constant, and the fluid is (almost) all gas. The resulting discontinuity in $\alpha$ at $z=b(t)$ implies that the limit $\delta \ll 1$ is singular, and there is a thin transition layer where $\alpha$ changes rapidly.

To study the transition layer near $b(t)$ we define

$$
z=b(t)+\delta Z \text {. }
$$

The governing equations become

$$
\begin{aligned}
\delta\left[\alpha_{t}-\frac{\dot{b}}{\delta} \alpha_{Z}+\frac{1}{\delta}(\alpha u)_{Z}\right] & =\Gamma, \\
-\alpha_{t}+\frac{\dot{b}}{\delta} \alpha_{Z}+\frac{1}{\delta}[(1-\alpha) u]_{Z} & =-\Gamma, \\
(1-\alpha)\left[p_{t}-\frac{\dot{b}}{\delta} p_{Z}+\frac{u}{\delta} p_{Z}\right] & =\Gamma\left(p-\frac{\Pi}{c_{0}}\right), \\
\frac{1}{\delta} p_{Z} & =-(1-\alpha),
\end{aligned}
$$


where $\dot{b}$ denotes the time derivative of $b(t)$. The boundary conditions at the bubble-point boundary are

$$
p=\Pi, \quad \alpha=0, \quad u=U(t), \quad \text { at } \quad Z=0 .
$$

Letting $\delta \rightarrow 0$, we see that (3.7d) gives $p=\Pi$. We can also solve for $\Gamma$ by substituting $(3.7 \mathrm{~d})$ into $(3.7 \mathrm{c})$ to get

$$
\Gamma=\frac{c_{0}(1-\alpha)^{2}(u-\dot{b})}{\Pi\left(1-c_{0}\right)} .
$$

We can then solve the first two equations (with $\delta \rightarrow 0$ ) to find the velocity

$$
u(Z, t)=\dot{b}-(\dot{b}-U(t)) \sqrt{1+\frac{2 Z c_{0}}{\Pi\left(1-c_{0}\right)}},
$$

and the gas volume fraction:

$$
\alpha(Z, t)=1-\frac{1}{\sqrt{1+\frac{2 Z c_{0}}{\Pi\left(1-c_{0}\right)}}} .
$$

The model predicts that at the bubble-point the multiphase flow converts almost instantaneously from a pure liquid to a highly gaseous foam, or else to a misty flow. The physical reason for this is that for any significant pressure below the bubble-point pressure, the equilibrium equation for the dissolved gas concentration indicates that a significant fraction of gas has been exsolved, and simple expansion necessitates that it must fill almost all the available volume.

This is contradictory to the oil-well literature which suggests that the oil domain supports gradual transitions between multiphase flow régimes (Shoham 2006, Al-Safran and Brill 2017), and that a significant liquid oil fraction exists at the outlet. The only way this can be consistent with the present homogeneous model is if the outlet pressure $p_{\text {out }} \approx p_{b}\left(p_{1} \approx \Pi\right.$ in dimensionless variables), but there is no reason that this should be the case, and it is generally not; for example, values quoted by Golan and Whitson (1991) for well No. B-11 in the Ekofisk oil field are $p_{\text {res }}=50 \mathrm{MPa}, p_{b}=36 \mathrm{MPa}$, and $p_{\text {out }}=29 \mathrm{MPa}$.

It is possible that this conclusion is a result of the assumption of equal phase velocities, which is due to the assumption (see (2.26)) that $\kappa_{D} \gg 1$, which itself requires sufficiently small bubbles (see table 2). As $\alpha$ grows, it is natural to suppose that this assumption breaks down, either through bubble growth or through régime transition, and that a two-fluid model becomes relevant. However, this would not alter the fact of rapid transition to a foam or mist, which is due to the fact that since $\delta \ll 1$ in (2.18a), then $\Gamma \ll 1$, so that (2.18c) again implies $p \approx$ constant, which requires $\alpha \approx 1$. We therefore keep the homogeneous model, but consider a different parameter régime, specifically that in which the initial concentration of dissolved gas is small; $c_{0} \sim \delta \ll 1$. It can be seen from (2.18c) that this is likely to spread out the exsolution region. 


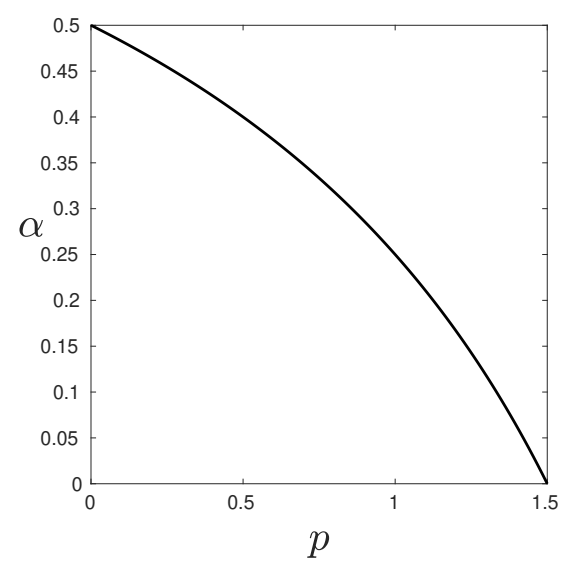

Figure 2.: The relationship between gas volume fraction and dimensionless pressure from (3.16), for varying dimensionless bubble-point pressures with $\Pi=1.5$ and $\lambda=1$.

\subsection{The case $c_{0} \sim \delta \ll 1$}

Let us suppose that $\delta / c_{0}=\lambda \sim O(1)$. We rescale $\Gamma=\delta \bar{\Gamma}$ (and then subsequently drop the overbar), assume $\delta \ll 1$, so that the equations (3.1a)-(3.1e) take the approximate form

$$
\begin{aligned}
\alpha_{t}+(\alpha u)_{z} & =\Gamma, \\
(1-\alpha)_{t}+[(1-\alpha) u]_{z} & =0, \\
{[(1-\alpha) c]_{t}+[(1-\alpha) c u]_{z} } & =-\lambda \Gamma, \\
p_{z} & =-(1-\alpha), \\
c & =\frac{p}{\Pi} .
\end{aligned}
$$

In the liquid region $0 \leq z \leq b(t)$ the results are the same as those derived above in section 3.1:

$$
p=p_{0}-z, \quad \alpha=0, \quad u=U(t), \quad b(t)=p_{0}-\Pi .
$$

For the two-phase region $b(t) \leq z \leq 1$, we utilise an observation by Fowler and Robinson (2018) that if two quantities $\phi$ and $\psi$ satisfy

$$
\begin{aligned}
\phi_{t}+(\phi u)_{z} & =0, \\
\psi_{t}+(\psi u)_{z} & =0,
\end{aligned}
$$

then

$$
\left(\frac{\phi}{\psi}\right)_{t}+u\left(\frac{\phi}{\psi}\right)_{z}=0 .
$$

If we sum $\lambda$ times (3.12a) and (3.12c) and then apply the above observation to the resultant equation and $(3.12 b)$, we find that $(c+\lambda \alpha /(1-\alpha))$ is advected with the flow. Using the fact that at $z=b(t), \alpha=0$ and $c=1$, this conserved quantity is in fact equal to one. Using (3.12e), we can write an expression for $\alpha$ in terms of $p$ :

$$
\alpha=\frac{1-\frac{p}{\Pi}}{1+\lambda-\frac{p}{\Pi}} .
$$

The gas volume fraction increases as the pressure decreases, see figure 2 , but now importantly $\alpha$ is bounded above by $1 /(1+\lambda)$. 


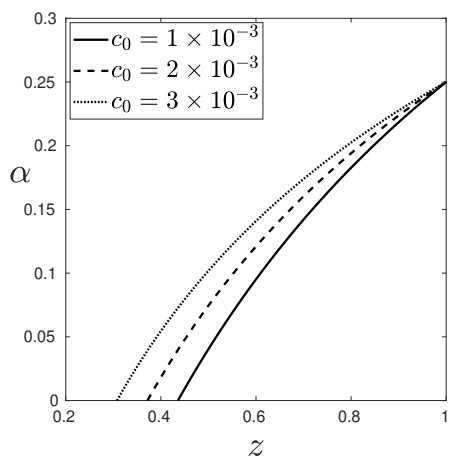

(a) $\alpha(z)$.

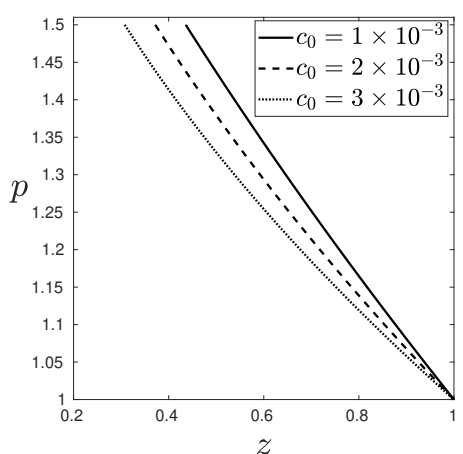

(b) $p(z)$.

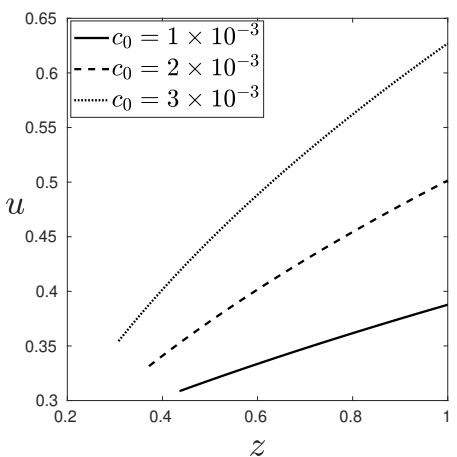

(c) $u(z)$.

Figure 3.: Analytical steady-state solutions to (3.12a)-(3.12e) using parameters from tables 1 and 2. For illustration purposes, we choose $\Pi=1.5, p_{1}=1$. Varying the value of $c_{0}$ gives different $p_{0}$ from (3.19) which in turns changes $b(t)$ from (3.3).

We solve the pressure-drop equation (3.12d) using (3.16) to obtain

$$
p=\Pi(1+\lambda)-\sqrt{\lambda^{2} \Pi^{2}+2 \lambda \Pi(z-b)} .
$$

The negative square root is selected, as this corresponds to a profile in which the pressure decreases with height.

The velocity profile can be found by using (3.12b) and (3.17) in (3.16) with the appropriate boundary conditions at $z=b(t)$, and the result of this is

$$
u=\dot{b}-[U(t)-\dot{b}]\left\{\frac{2(z-b)+\lambda \Pi}{\lambda \Pi}\right\}^{1 / 2} .
$$

Applying the boundary condition for the pressure at $z=1$ in (3.17), with the definition of $b(t)$ from (3.3), we obtain

$$
p_{0}=\left(1+p_{1}\right)-\frac{\left(p_{1}-\Pi\right)^{2}}{2 \lambda \Pi},
$$

and this determines the inlet velocity, since from (2.22a), $U=1-\delta_{c} p_{0}$.

The steady-state analytical solutions $\alpha$ (3.16), $p$ (3.17), and $u$ (3.18) are shown in figure 3 . We used the parameters given in tables 1 and 2 , and examined the effect of changing the initial concentration of dissolved gas $c_{0}$, which affects the inflow pressure $p_{0}$ from (3.19) and the value for $b$.

Increasing $c_{0}$ resulted in a shorter single-phase domain i. e. reduced $b$, see figure 3 , which suggests that for higher initial gas concentrations, the pressure gradient from the inlet pressure to the bubble-point pressure is increased. The exit pressure $p_{1}$ is a boundary condition in the system, so for changing $c_{0}$ the pressure drop was the same but over a different domain, see figure $3 \mathrm{~b}$. As the exit pressure was fixed, the exit volume fraction was also fixed due to (3.16), so the same amount of gas was released but over changing domain lengths, see figure 3a. Moreover, increasing $c_{0}$ corresponded to an increased velocity, see figure 3c.

This analysis shows that by assuming that the initial amount of dissolved gas to be small and comparable to the density ratio, we can essentially stretch out the two-phase flow region. This parameter limit is appropriate for oil wells in producing fields such as Reshadat, discussed in section 3. However, in general it will not always be true that the dissolved gas fractions will be small. We will now explore another possible mechanism to prevent immediate gasification, and that is the existence of rate limiting non-equilibrium exsolution dynamics. 


\section{Non-equilibrium dynamics}

The prescribed Henry's law (2.10) assumes equilibrium between the concentration of dissolved gas and the bulk pressure. However, there are various ways by which the system may not be in equilibrium. The first is that, although the liquid-gas surface may be at equilibrium, in general the bulk dissolved gas concentration in the liquid is super-saturated, in that the dissolved concentration is higher than an equilibrium concentration. When the pressure drops below the bubble-point, the dissolved gas is able to come out of solution and nucleate finite radius bubbles which then grow due to the diffusion of the gas in solution. Including the nucleation and bubble growth kinetics should be rate limiting for the exsolution of gas. We will consider the two issues in turn.

\subsection{Diffusion of dissolved gas}

Suppose that at $z=b(t)$ small bubbles appear and then grow due to diffusion of dissolved gas from the liquid to the bubble interface. Let $n$ be the number density, by volume, of bubbles. If the typical distance between the bubbles is $L_{b}$, then we have $n=1 / L_{b}^{3}$. If $a_{b}$ is the bubble radius and we assume spherical bubbles then the bubble volume $V_{b}$ is

$$
V_{b}=\frac{4 \pi a_{b}^{3}}{3} .
$$

Note that

$$
\alpha=n V_{b}=\frac{4 \pi a_{b}^{3}}{3 L_{b}^{3}} .
$$

We assume that $L_{b} \gg a_{b}$, and consider a domain centred at a bubble with spherical polar radius $r$. Diffusion of gas to a single isolated bubble is given by the diffusion equation

$$
\begin{aligned}
c_{t} & =D \nabla^{2} c, \\
c & =c_{\text {sat }} \text { at } r=a_{b}, \\
c & \rightarrow \bar{c} \quad \text { as } r \rightarrow \infty,
\end{aligned}
$$

where $c$ is the concentration of dissolved gas, $D$ is the diffusion coefficient, $c_{\text {sat }}$ is the saturated concentration and $\bar{c}$ is the far-field supersaturated concentration.

The time scale of the large scale flow, from (2.16) and table 1 , is $t_{f}=L / U_{0} \sim 0.5 \times 10^{4} \mathrm{~s}$. If we scale (4.3a) with typical bubble radius $a_{0}\left(r \sim a_{b} \sim a_{0}\right)$ then $t_{d}=a_{0}^{2} / D$ is the diffusive time scale. Note that the Reynolds number $R e=\rho_{l} U_{0} d / \mu \gg 1$, so it is realistic to assume a turbulent flow and consequently take $D \approx \varepsilon_{T} U_{0} d$, where a typical value of $\varepsilon_{T}$ depends on the Reynolds number $R e$ and is $\varepsilon_{T} \approx 0.001$ for $R e \sim 10^{4}$ (Fowler 2011). This yields $D \sim 2 \times 10^{-5}$ $\mathrm{m}^{2} \mathrm{~s}^{-1}$. If we consider small bubbles with $a_{0}=1 \mathrm{~mm}$, then $t_{d} \sim 0.05 \mathrm{~s}$, so we may reasonably take $t_{d} \ll t_{f}$ and (4.3a) becomes $\nabla^{2} c=0$, which we solve using the boundary conditions (4.3b) and (4.3c), thus

$$
c=\bar{c}+\frac{\left(c_{\mathrm{sat}}-\bar{c}\right) a_{b}}{r} .
$$

The resulting diffusive flux of gas across the bubble boundary provides the following constitutive equation for bubble growth:

$$
\dot{a}_{b}=\frac{D}{a_{b}}\left(\bar{c}-c_{\mathrm{sat}}\right),
$$

where $\dot{a}_{b}$ is the material time derivative following the bubbles. This theory is due to Lifshitz and Slyozov (1961). The bubbles will therefore stop growing when equilibrium at $\bar{c}=c_{\mathrm{sat}}$ is 
reached. (4.5) can be translated into an equation for $\alpha$ using (4.1) and (4.2) and assuming the number density $n$ is fixed; this yields

$$
\alpha_{t}+u \alpha_{z}=(4 \pi n)^{2 / 3}(3 \alpha)^{1 / 3} D\left(\bar{c}-c_{\mathrm{sat}}\right) .
$$

With the assumption that the bubbles are far apart, we can take the far-field concentration to be the average concentration; $\bar{c}=c$. Furthermore the saturated concentration is proportional to the pressure as in Henry's law, $c_{\text {sat }}=K p$ in dimensional form. (4.6) therefore replaces the Henry's law equilibrium in which the right-hand side is zero.

If we scale the variables as in (2.16), the dimensionless form of (4.6) is

$$
\frac{t_{g}}{t_{f}}\left[\alpha_{t}+u \alpha_{z}\right]=\left(48 \pi^{2} \alpha\right)^{1 / 3}\left(c-\frac{p}{\Pi}\right),
$$

where the associated bubble growth time scale $t_{g}=1 /\left(D c_{0} n^{2 / 3}\right)=L_{b}^{2} /\left(D c_{0}\right)$. Our earlier assumption of equilibrium is therefore valid when $t_{g} \ll\left(48 \pi^{2}\right)^{1 / 3} t_{f} \approx 7.8 t_{f}$, and this is true if

$$
n \gg n^{*} \equiv \frac{1}{4 \pi \sqrt{3}}\left(\frac{U_{0}}{L D c_{0}}\right)^{3 / 2} \approx 0.046\left(\frac{U_{0}}{L D c_{0}}\right)^{3 / 2},
$$

and thus the assumption of equilibrium solubility rests on the number of bubbles that are nucleated. Note that our earlier assumption that $t_{d} \ll t_{f}$ is essentially equivalent to $t_{g} \ll t_{f}$, so that in conditions of non-equilibrium, where $n<n^{*}$, the Lifshits-Slyozov theory can not accurately be applied.

Using the turbulent diffusivity $D \sim 2 \times 10^{-5} \mathrm{~m}^{2} \mathrm{~s}^{-1}$ together with the parameter values in table 1 and the assumption that $c_{0}=0.2$ implies $n^{*}=16.3 \mathrm{~m}^{-3}$ in (4.8), and we conclude that diffusion is not a limiting factor. So now we turn to the issue of nucleation itself.

\subsection{Nucleating bubbles}

We now consider a population of bubbles whose number density is a function of their size. For convenience we choose the size variable to be the bubble surface area $s=4 \pi a_{b}^{2}$, and denote the bubble number density as $n(s, z, t)$. The number density of bubbles per unit volume with surface area $s$ is $n(s, z, t) \mathrm{d} s$. The number density of bubbles per unit volume is thus given by

$$
N(z, t)=\int_{0}^{\infty} n \mathrm{~d} s .
$$

The gas volume fraction is given by

$$
\alpha=\frac{1}{6 \sqrt{\pi}} \int_{0}^{\infty} s^{3 / 2} n \mathrm{~d} s .
$$

Conservation of the bubble size distribution is given by

$$
n_{t}+(n u)_{z}+(n v)_{s}=0,
$$

where $v$ is the bubble growth rate. From (4.5) the bubble growth rate is given by

$$
v=8 \pi D\left(c-c_{\mathrm{sat}}\right),
$$

(dropping the overbar on $\bar{c}$ ).

We can presume $n \rightarrow 0$ as $s \rightarrow \infty$; we also need to prescribe a boundary condition at $s=0$, $\left.n\right|_{s=0}=n_{0}$. Integration of (4.11) yields

$$
N_{t}+(N u)_{z}=\left[8 \pi D\left(c-c_{\mathrm{sat}}\right)\right] n_{0} .
$$


If we assume that the rate of nucleation is proportional to the supersaturation of dissolved gas (which we now assume to be out of equilibrium, i. e. $c>c_{\text {sat }}$ ), then we can write

$$
N_{t}+(N u)_{z}=k_{N}\left(c-c_{\mathrm{sat}}\right),
$$

where $k_{N}$ is a prescribed rate constant, having units of $\mathrm{m}^{-3} \mathrm{~s}^{-1}$. Using this in (4.13) provides the boundary condition for $n$ :

$$
n=n_{0}=\frac{k_{N}}{8 \pi D} \text { at } s=0 .
$$

Multiplying (4.11) by $s^{3 / 2} /(6 \sqrt{\pi})$ and integrating gives, after integrating the last term by parts,

$$
\alpha_{t}+(\alpha u)_{z}=2 \sqrt{\pi} D\left(c-c_{\mathrm{sat}}\right) \int_{0}^{\infty} s^{1 / 2} n \mathrm{~d} s .
$$

The homogeneous model we derived earlier is now modified by the nucleation equation (4.11) and its consequence (4.16). The boundary conditions are also as in (2.22a)-(2.22c) but with the additional condition (4.15). The model is made dimensionless as earlier in (2.16) (but with the modification that $\Gamma \sim \rho_{g} U_{0} / L$, as introduced at the beginning of section 3.2), and additionally

$$
n \sim n_{0}=\frac{k_{N}}{8 \pi D}, \quad s \sim\left(\frac{6 \sqrt{\pi}}{n_{0}}\right)^{2 / 5} ;
$$

we then obtain the dimensionless model

$$
\begin{aligned}
\alpha_{t}+(\alpha u)_{z} & =\Gamma, \\
(1-\alpha)_{t}+[(1-\alpha) u]_{z} & =-\delta \Gamma, \\
{[(1-\alpha) c]_{t}+[(1-\alpha) c u]_{z} } & =-\frac{\delta}{c_{0}} \Gamma, \\
F^{2}[(1-\alpha)+\delta \alpha]\left[u_{t}+u u_{z}\right] & =-p_{z}-\kappa_{w} u^{2}-[(1-\alpha)+\delta \alpha], \\
\alpha_{t}+(\alpha u)_{z} & =\gamma\left(c-\frac{p}{\Pi}\right) \int_{0}^{\infty} s^{1 / 2} n \mathrm{~d} s, \\
n_{t}+(n u)_{z}+\frac{2 \gamma}{3}\left(c-\frac{p}{\Pi}\right) n_{s} & =0,
\end{aligned}
$$

where the parameters are those defined in (2.19), and we have introduced the dimensionless parameter

$$
\gamma=\frac{2 L c_{0}}{U_{0}}\left(\frac{27}{8} \pi^{2} k_{N}^{2} D^{3}\right)^{1 / 5} .
$$

(4.18e) is implied by (4.18f) together with the scaled version of (4.10):

$$
\alpha=\int_{0}^{\infty} s^{3 / 2} n \mathrm{~d} s .
$$

The boundary conditions for these equations are

$$
\begin{aligned}
\alpha=0, \quad c=1, \quad p=p_{0}, \quad u=U(t)=1-\delta_{c} p_{0} & \text { at } z=0, \\
\alpha=0, \quad c=1, \quad p=\Pi, \quad n=0 & \text { at } z=b(t), \\
p=p_{1} & \text { at } z=1, \\
n=1 & \text { at } s=0 .
\end{aligned}
$$


(4.18a) and (4.18e) give an expression for the mass-transfer rate $\Gamma$ :

$$
\Gamma=\gamma\left(c-\frac{p}{\Pi}\right) \int_{0}^{\infty} s^{1 / 2} n \mathrm{~d} s
$$

In the single-phase region $0 \leq z \leq b(t)$ our earlier results still hold. We therefore analyse the two-phase region $b(t) \leq z \leq 1$. Again we take $F^{2}, \kappa_{w}, \delta \ll c_{0} \sim 1$, and consider different parameter limits for $\gamma$.

4.2.1. $\gamma \gg 1$

From the definition (4.19), large $\gamma \gg 1$ corresponds to a large nucleation rate $k_{N}$. If we take $\gamma \gg 1$ and neglect other small terms, the equations to solve become:

$$
\begin{aligned}
\alpha_{t}+(\alpha u)_{z} & =\gamma\left(c-\frac{p}{\Pi}\right) \int_{0}^{\infty} s^{1 / 2} n \mathrm{~d} s \\
(1-\alpha)_{t}+((1-\alpha) u)_{z} & =0 \\
((1-\alpha) c)_{t}+((1-\alpha) c u)_{z} & =0 \\
p_{z} & =-(1-\alpha), \\
n_{t}+(n u)_{z}+\frac{2 \gamma}{3}\left(c-\frac{p}{\Pi}\right) n_{s} & =0 .
\end{aligned}
$$

Now as in (3.15), (4.23b) and (4.23c) imply that $c=1$ is advected with the flow. Furthermore (4.23a) gives that $c=p / \Pi$, the dissolved concentration is at equilibrium, and we have again that $p=\Pi$. From (4.23d) this implies $\alpha=1$, which in (4.23b) gives $u=U(t)$. The model conclusions are exactly the same as in section 3.1; above the bubble-point pressure at $z=b(t)$ the mixture is mostly gas.

\subsection{2. $\gamma \ll 1$}

If we consider small $\gamma \ll 1$, i. e. small nucleation rate, then the model equations are approximately

$$
\begin{aligned}
\alpha_{t}+(\alpha u)_{z} & =0 \\
(1-\alpha)_{t}+((1-\alpha) u)_{z} & =0 \\
((1-\alpha) c)_{t}+((1-\alpha) c u)_{z} & =0 \\
p_{z} & =-(1-\alpha), \\
n_{t}+(n u)_{z} & =0 .
\end{aligned}
$$

If we sum (4.24a) and (4.24b) we get $u=U(t)$. Again, (4.24b) and (4.24c) imply that $c=1$ is advected with the flow. Now we also have a constant gas volume fraction $\alpha=0$ being advected with the flow, from (4.24a). Using this in (4.24d) gives a linearly decreasing pressure $p=\Pi-z$. The model solutions are again trivial, but the conclusion for small $\gamma$ is that the gas volume fraction remains small throughout the pipe.

An item of interest is how the bubble number density behaves. Solving the equation for $n$ indicates that $n=1$ for $s=0$, but $n=0$ for $s>0$. The discontinuity is resolved by a boundary layer when $s \sim \gamma$, as can be seen from (4.18f).

\subsection{3. $\gamma \sim O(1)$}

For large $\gamma \gg 1$ we have mostly gas at the top of the well, and for small $\gamma \ll 1$ we have mostly liquid. We now consider the case $\gamma \sim O(1)$, where in the $\delta \ll c_{0} \sim 1$ limit, the system 
of equations is approximately

$$
\begin{aligned}
\alpha_{t}+(\alpha u)_{z}=\Gamma & =\gamma\left(c-\frac{p}{\Pi}\right) \int_{0}^{\infty} s^{1 / 2} n \mathrm{~d} s, \\
(1-\alpha)_{t}+((1-\alpha) u)_{z} & =0, \\
((1-\alpha) c)_{t}+((1-\alpha) c u)_{z} & =0, \\
p_{z} & =-(1-\alpha), \\
n_{t}+(n u)_{z}+\frac{2 \gamma}{3}\left(c-\frac{p}{\Pi}\right) n_{s} & =0 .
\end{aligned}
$$

Note that $(4.25 \mathrm{~b})$ and $(4.25 \mathrm{c})$ again imply that $c \approx 1$ is advected with the flow. From $(4.25 \mathrm{a})$ and (4.25b), $u_{z}=\Gamma$. Further, we can integrate (4.25d) to obtain $p$ as

$$
p=\Pi-\int_{b}^{z}[1-\alpha(y, t)] \mathrm{d} y,
$$

and thus

$$
\left(1-\frac{p}{\Pi}\right)=\frac{1}{\Pi} \int_{b}^{z}(1-\alpha) \mathrm{d} y, \quad \Gamma=u_{z}=\frac{\gamma}{\Pi} \int_{b}^{z}(1-\alpha) \mathrm{d} y \int_{0}^{\infty} s^{1 / 2} n \mathrm{~d} s .
$$

The equation for $n$ takes the form

$$
n_{t}+u n_{z}+\frac{2 \gamma}{3}\left(1-\frac{p}{\Pi}\right) n_{s}=-u_{z} n
$$

together with boundary conditions

$$
\begin{aligned}
& n=1 \text { on } s=0, \\
& n=0 \text { on } z=b(t) .
\end{aligned}
$$

(There is also an initial condition, whose effect is washed out of the system after a finite time.) Using the definition of $\alpha$ in (4.20), this becomes an equation in terms of $n(z, s, t)$ alone.

Note that $b(t)$ and $p_{0}$ (and thus $\left.U(t)\right)$ in the boundary conditions $(4.21 \mathrm{a})-(4.21 \mathrm{~b})$ are not known. Applying $p=p_{1}$ at $z=1$ in (4.26) provides an equation for $b(t)$, and then $p_{0}$ is determined from (3.3).

To solve the steady state version of (4.28) numerically we applied a change of variables $\hat{z}=$ $\frac{z-b}{1-b}$ to remove $b$ from the integral limits in (4.26)-(4.27). The solution domain is consequently $\hat{z} \in[0,1]$. We then used an implicit-explicit finite difference scheme for $n(z, s)$ and a shooting method for the unknown $b$. The number density of bubbles $N$ and gas volume fraction $\alpha$ can be calculated from $n$ using (4.9) and (4.20) respectively. We present the numerical solutions, using parameters from tables 2 , in figure 4 for different values of $\gamma$.

For large $\gamma$, the results predict a larger volume fraction $\alpha$, and a higher number of bubbles $N$. The gas volume fraction profiles in figure $4 \mathrm{a}$ support the conclusion from the parameter limit $\gamma \gg 1$ in section 4.2.1 that increasing $\gamma$ corresponds to increased gas in the pipe. We can investigate this further by looking at the gas volume fraction at the top of the pipe as a function of $\gamma$. The model predicts an increasing exit volume fraction which tends to $\alpha=0.9$, see figure 5. It is clear that the value that the exit volume fraction tends to is dependent on the choice of parameters, namely the exit pressure.

The number density of bubbles per unit volume is given by $N$. We rescale the numerical solutions according to plot the dimensional number density $N$ in figure $4 \mathrm{~b}$. We see that for larger $\gamma$ we have more bubbles. These results suggest that for larger $\gamma$ there are lots of small bubbles in the pipe corresponding to a foam-like mixture.

We see that for $\gamma \sim O(1)$ the addition of non-equilibrium dynamics to the two-phase flow equations yields a two-phase flow in which the gas volume fraction increases over the length 


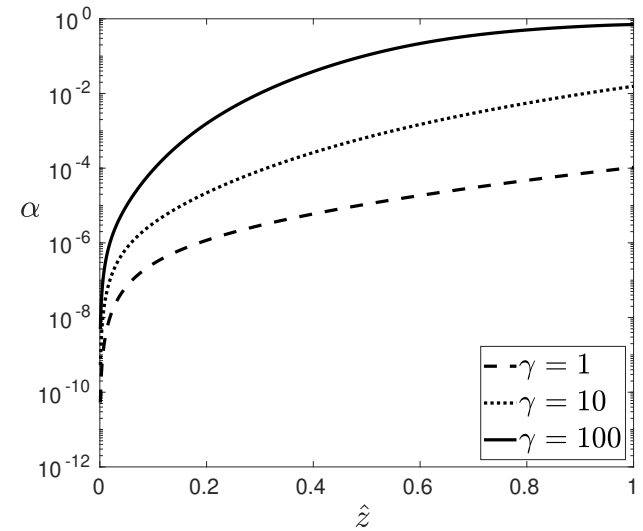

(a) $\alpha(\hat{z})$.

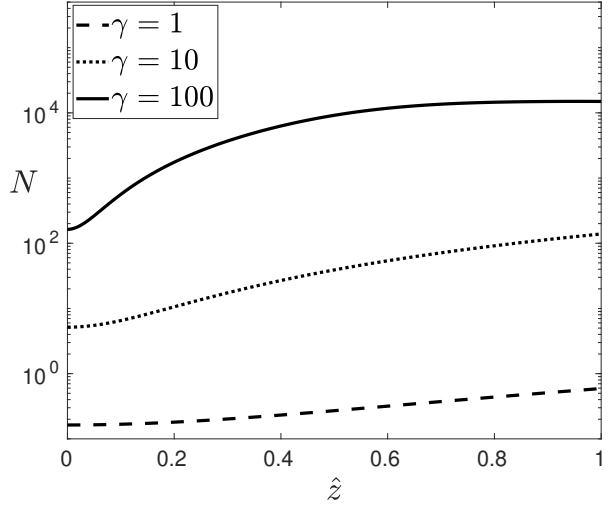

(b) $N(\hat{z})$.

Figure 4.: Steady-state solutions for the gas volume fraction $\alpha$ and number density of bubbles $N\left(\mathrm{~m}^{-3}\right)$ for various values of $\gamma$ with parameters from tables 1 and 2 , and $\Pi=1.5, p_{1}=1$. Prescribing the value of $\gamma$ provides values for the nucleation rate constant $k_{N}$ from (4.19). For $\gamma=1,10,100$ and $k_{N}=0.01,3.4,1082.9 \mathrm{~m}^{-3} \mathrm{~s}^{-1}$ respectively.

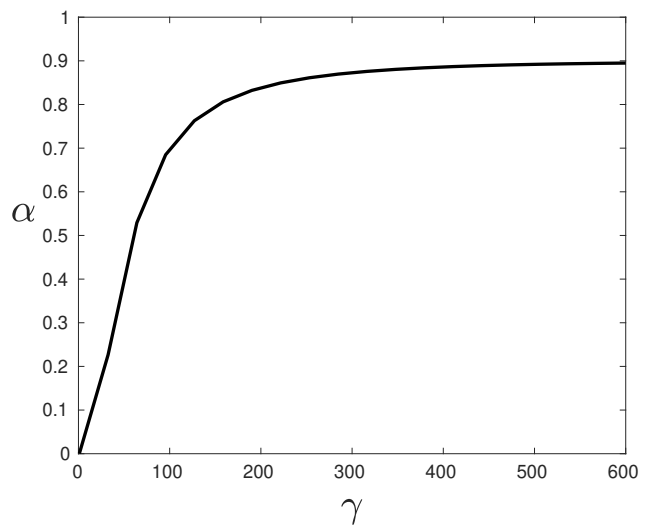

Figure 5.: The exit volume fraction $\alpha(z=1)$ as a function of $\gamma$ with parameters from tables 1 and 2 , and $\Pi=1.5, p_{1}=1$.

of the tube, with an increasing outflow volume fraction for increasing $\gamma$.

\section{Conclusions}

This paper formulates a homogeneous model for the upwelling two-phase flow in oil wells. Previous oil well models do not consider the conservation of mass for the gas dissolved in the liquid phase as well as the gas that is a distinct phase itself. The novelty in the model derived in this paper is that there are three mass conservation equations; one each for the liquid phase, the total gas phase, and the dissolved gas in the liquid. The theory behind the model was motivated by noting the similarity between upwelling flow in oil wells and volcanic eruptions, and we utilised recent work by Fowler and Robinson (2018).

This simple model of two incompressible fluids produced an unexpected rapid change from liquid to a highly gaseous fluid at the bubble-point pressure. We found that if the gas to liquid density ratio $\delta$ is small, the model is singularly perturbed. By examining the boundary 
layer at the bubble-point pressure boundary, we derived analytical solutions to the model equations. A predominantly gaseous outflow is unexpected as the oil literature commonly describes two-phase producing wells. To our knowledge, no previous model has observed the dramatic change from liquid to highly gaseous mixtures at the bubble-point.

In order to find a more realistic solution in which the transition zone is elongated, so that a two-phase mixture is produced at the outlet, we can suppose that the initial amount of dissolved gas $c_{0}$ is proportional to the density ratio $\delta$. However, this constraint will not generally be satisfied. We then considered the effects of non-equilibrium dynamics. Firstly, Henry's law was modified to allow for supersaturation of the dissolved gas phase: this made little difference to the solution, as diffusion through the liquid was found to be rapid. Secondly, a model for nucleation and bubble growth was formulated, and the solutions were found to depend critically on the nucleation rate; if this is sufficiently low, then exsolution is diminished significantly.

In more detail, it was found that gas formation is suppressed ( $\alpha<1$ at the outlet) if a dimensionless parameter $\gamma$ given by (4.19) is less than a critical value $\gamma_{c} \approx 70$; we take the value where $\alpha=0.5$ in figure 5 . Rewriting this in terms of the nucleation rate constant $k_{N}$ defined through (4.14), we find that a two-phase mixture at the outlet requires

$$
k_{N}<k_{c}=\frac{1}{\pi}\left(\frac{2}{3 D}\right)^{3 / 2}\left(\frac{\gamma_{c} U_{0}}{2 L c_{0}}\right)^{5 / 2} .
$$

Using the values $D=2 \times 10^{-5} \mathrm{~m}^{2} \mathrm{~s}^{-1}, U_{0}=0.2 \mathrm{~m} \mathrm{~s}^{-1}, L=10^{3} \mathrm{~m}$ and $c_{0}=0.2$, this gives $k_{c} \approx 444 \mathrm{~m}^{-3} \mathrm{~s}^{-1} \approx 0.4 \times 10^{-3} \mathrm{~cm}^{-3} \mathrm{~s}^{-1}$. Unfortunately, little is known about the nucleation characteristics of the flows in oil wells.

In the model presented in this paper, we consider governing equations relevant for a bubbly flow characterised by a sufficiently small gas volume fraction. However, as the bubbles nucleate and grow, additional bubble dynamics including coalescence and break-up will occur. When the gas volume fraction is sufficiently large, the flow will undergo a régime transition from bubbly to slug flow. In slug flow the slip between phases and the wall stress of the gas becomes important. However, the main conclusions of the model remain unchanged as the prediction of an instantaneous increase in gas volume fraction is driven by the decompression of the gas and the prescribed relationship between pressure and dissolved gas concentration in Henry's law (2.10). The examination of the effect of coalescence dynamics and the bubbly-to-slug flow régime transition will be important for the $\delta / c_{0} \sim O(1)$ system in section 3.2, and is reserved for future work.

In this paper we consider incompressible-incompressible two-phase flow. We note that the gaseous phase in oil wells is, in fact, highly compressible. However, the inclusion of compressibility effects would not alter our conclusion; the gas density will vary but the density ratio $\delta$ will always be expected to be small, and it is this key parameter that is driving our model conclusions. For a more detailed examination of the compressible-incompressible model see Pereira (2019).

The results of this paper suggest it may be important to include nucleation and growth kinetics when modelling upwelling flow in oil wells. To our knowledge, this approach is not used in commercial oil well models. Such models rely heavily on empirically derived correlations between unknown parameters and variables. The work presented in this paper provides a more deterministic approach.

\section{Acknowledgements}

This publication is based on work supported by the EPSRC Centre For Doctoral Training in Industrially Focused Mathematical Modelling (EP/L015803/1) in collaboration with 
Petrotechnical Data Systems. A. C. F. acknowledges the support of the Mathematics Applications Consortium for Science and Industry (www.macsi.ul.ie) funded by the Science Foundation Ireland mathematics grant 12/IA/1683.

\section{References}

Al-Safran, E.M. and Brill, J.P., Applied Multiphase Flow in Pipes and Flow Assurance - Oil and Gas Production, 2017 (Society of Petroleum Engineers Richardson, TX).

Ansari, A.M., Sylvester, N.D., Shoham, O. and Brill, J.P., A comprehensive mechanistic model for upward two-phase flow in wellbores; in SPE Annual Technical Conference and Exhibition, 1990.

Beggs, D.H. and Brill, J.P., A study of two-phase flow in inclined pipes. Journal of Petroleum technology, 1973, 25, 607-617.

Bendiksen, K.H., Maines, D., Moe, R. and Nuland, S., The dynamic two-fluid model OLGA: Theory and application. SPE Production Engineering, 1991, 6, 171-180.

Cazarez-Candia, O. and Vásquez-Cruz, M.A., Prediction of pressure, temperature, and velocity distribution of two-phase flow in oil wells. Journal of Petroleum Science and Engineering, 2005, 46, 195-208.

Chen, Z., Reservoir simulation: mathematical techniques in oil recovery, Vol. 77, 2007 (SIAM).

Christensen, N.I. and Mooney, W.D., Seismic velocity structure and composition of the continental crust: A global view. Journal of Geophysical Research: Solid Earth, 1995, 100, 9761-9788.

Dale, B.H., Production Optimization Using NODAL Analysis, 1991 (Oil and Gas Consultants international publications, Tulsa, Oklahoma)

Drew, D.A. and Passman, S.L., Theory of multicomponent fluids, Vol. 135, 2006 (Springer Science \& Business Media).

Duns Jr, H. and Ros, N.C.J., Vertical flow of gas and liquid mixtures in wells; in 6th World Petroleum Congress, 1963.

Economides, M.J., Petroleum production systems, 2013 (Upper Saddle River).

Fowler, A.C., Mathematical geoscience, Vol. 36, 2011 (Springer Science \& Business Media).

Fowler, A.C. and Robinson, M., Counter-current convection in a volcanic conduit. Journal of Volcanology and Geothermal Research, 2018, 356, 141-162.

Ghorbani, H., Wood, D.A., Moghadasi, J., Choubineh, A., Abdizadeh, P. and Mohamadian, N., Predicting liquid flow-rate performance through wellhead chokes with genetic and solver optimizers: an oil field case study. Journal of Petroleum Exploration and Production Technology, 2018, pp. 1-19.

Golan, M. and Whitson, C.H., Well performance, 1991 (Prentice Hall PTR, 2nd Revised edition).

Govier, G.W. and Aziz, K., The flow of complex mixtures in pipes, 2008 (Society of Petroleum Engineers).

Guo, B., Petroleum production engineering, a computer-assisted approach, 2011 (Gulf Professional Publishing).

Hagedorn, A.R. and Brown, K.E., Experimental study of pressure gradients occurring during continuous twophase flow in small-diameter vertical conduits. Journal of Petroleum Technology, 1965, 17, 475-484.

Ishii, M. and Mishima, K., Two-fluid model and hydrodynamic constitutive relations. Nuclear Engineering and design, 1984, 82, 107-126.

Lifshitz, I.M. and Slyozov, V.V., The kinetics of precipitation from supersaturated solid solutions. Journal of physics and chemistry of solids, 1961, 19, 35-50.

Lisseter, P.E. and Fowler, A.C., Bubbly flow - A simplified model. International Journal of Multiphase Flow, 1992, 18, 195-204.

Livescu, S., Durlofsky, L.J., Aziz, K. and Ginestra, J.C., A fully-coupled thermal multiphase wellbore flow model for use in reservoir simulation. Journal of Petroleum Science and Engineering, 2010, 71, $138-146$.

Meyer, R.F. and Attanasi, E.D., Heavy oil and natural bitumen-strategic petroleum resources. 2003, Technical report, United States Geological Survey.

Muggeridge, A., Cockin, A., Webb, K., Frampton, H., Collins, I., Moulds, T. and Salino, P., Recovery rates, enhanced oil recovery and technological limits. Philosophical Transactions of The Royal Society A, 2014, 372.

Pauchon, C. and Banerjee, S., Interphase momentum interaction effects in the averaged multifield model: Part I: Void propagation in bubbly flows. International journal of multiphase flow, 1986, 12, 559-573.

Pereira, V.E., Multiphase pipe-flow with an application to oil wells. Ph.D. Thesis, University of Oxford, 2019.

Poettman, F.H. and Carpenter, P.G., The multiphase flow of gas, oil, and water through vertical flow strings with application to the design of gas-lift installations; in Drilling and Production Practice, 1952.

Shippen, M. and Bailey, W.J., Steady-State Multiphase Flow Past, Present, and Future, with a Perspective on Flow Assurance. Energy \& Fuels, 2012, 26, 4145-4157.

Shoham, O., Mechanistic Modeling of Gas-Liquid Two-Phase Flow in Pipes, 2006 (Society of Petroleum Engineers Richardson, TX).

Stuhmiller, J.H., The influence of interfacial pressure forces on the character of two-phase flow model equations. International Journal of Multiphase Flow, 1977, 3, 551-560.

Taitel, Y. and Dukler, A.E., A model for predicting flow regime transitions in horizontal and near horizontal gas-liquid flow. AIChE Journal, 1976, 22, 47-55. 
Van Den Bark, E. and Thomas, O.D., Ekofisk: first of the giant oil fields in Western Europe. AAPG Bulletin, 1981, 65, 2341-2363. Vazquez, M. and Beggs, H.D., Correlations for fluid physical property prediction; in SPE Annual Fall Technical Conference and Exhibition, 1977.

Wallis, G.B., One-dimensional two-phase flow, 1969 (McGraw-Hill).

Wals, G.B., One-dinensional tuo-phase flow, 1969 (McGraw-Hill).

exsolve final Dynamics
REFERENCES
O.D., Ekofisk: first of the giant oil fields in Western Europe. AAPG Bulletin,
orrelations for fluid physical property prediction; in SPE Annual Fall Technical
977.

.

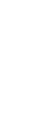

